\title{
Médiévales
}

Langues, Textes, Histoire

75 | automne 2018

Traductions du Moyen Âge

\section{La littéracie des femmes à la fin du Moyen Âge. Questions sur l'histoire de la culture, de la lecture et de l'écriture à travers des travaux récents}

\section{Sylvie Duval}

\section{(2) OpenEdition Journals \\ Édition électronique \\ URL : https://journals.openedition.org/medievales/9559 \\ DOI : 10.4000/medievales.9559 \\ ISSN : $1777-5892$ \\ Éditeur \\ Presses universitaires de Vincennes}

Édition imprimée

Date de publication : 15 octobre 2018

Pagination : 227-248

ISBN : 978-2-84292-861-2

ISSN : 0751-2708

Référence électronique

Sylvie Duval, «La littéracie des femmes à la fin du Moyen Âge. Questions sur l'histoire de la culture, de la lecture et de l'écriture à travers des travaux récents », Médiévales [En ligne], 75 | automne 2018, mis en ligne le 15 octobre 2019, consulté le 22 avril 2022. URL : http://journals.openedition.org/ medievales/9559; DOI : https://doi.org/10.4000/medievales.9559 
Sylvie Duval

\section{La littéracie des femmes à la fin du Moyen Âge Questions sur l'histoire de la culture, de la lecture et de l'écriture à travers des travaux récents}

Le mot littéracie est un anglicisme dont l'emploi est devenu fréquent dans les sciences humaines. Traduisible par «alphabétisation », mais aussi plus largement par « connaissance des lettres », il se réfère, pour les chercheurs, à une conception ouverte et dynamique de l'approche de l'écrit, depuis la simple capacité à déchiffrer quelques mots jusqu'à la possibilité de composer et de rédiger entièrement un texte complexe, voire à le faire en plusieurs langues ${ }^{1}$. La diffusion de l'usage de ce mot et du concept qu'il recoupe, forgé par les chercheurs anglo-américains dans les années 1970, reflète les évolutions récentes à propos de la façon dont on envisage la capacité à lire et à écrire de populations données, et surtout le besoin de dépasser une vision trop rigide et limitée de cette capacité (être alphabétisé ou ne pas l'être). L'histoire médiévale participe activement de ce renouvellement épistémologique. Il existe une tradition déjà longue, chez les médiévistes, de l'histoire des "pratiques de l'écrit », liée à des questions propres à leur période d'étude : celle des rapports entre oralité et scripturalité et des usages respectifs du latin et des langues vernaculaires, celle de l'émergence d'une ou de plusieurs littérature(s) européennes, celle, enfin, d'une différence entre un savoir clérical et savant et un savoir « laïc ». Ces questions transversales trouvent aujourd'hui de nouvelles interprétations grâce à l'idée de littéracie, dont l'association avec le concept du genre est susceptible d'ouvrir de nouvelles perspectives de recherches.

1. Sur l'emploi du concept de « litéracie » ou « littéracie » en France, voir notamment C. Brissaud, C. Barre de Miniac, M. Rispail éd., La Littéracie. Conceptions théoriques et pratiques d'enseignement de la lecture-écriture, Paris, 2004. On trouve aussi parfois « alphabétisme ». J'utiliserai, tout au long de cet article, la forme « littéracie », simplement pour me conformer à l'usage qui m'a paru être le plus courant. 


\section{La littéracie en histoire médiévale : un bref aperçu}

En 1939, James Westphall Thompson assimilait, dans son ouvrage intitulé The Literacy of the Laity in the Middle Ages, les lettrés médiévaux aux personnes capables de lire et de comprendre le latin ${ }^{2}$, suivant ainsi la définition médiévale du clericus litteratus. Cette approche n'est plus d'actualité aujourd'hui, ainsi que le rappelle Paul Bertrand dans un ouvrage récent :

Il faut faire un sort au discours manichéen séparant les litterati, les «lettrés », et les analphabètes : nombre de cultures de l'écrit coexistent au sein de cette société médiévale, depuis celle de l'homme qui peut lire un petit texte mais est rétif à l'écriture de plus de cinq lignes à celle du technicien des comptes qui jongle avec les chiffres mais écrit malhabilement ${ }^{3}$.

Cette remise en question, déjà ancienne ${ }^{4}$, a surtout été le fait d'historiens anglo-américains, tels Malcolm Parkes ${ }^{5}$ ou Michael Clanchy, dont l'ouvrage From Memory to Written Record (1979), qui mit en relation le grand développement des écritures pratiques avec un accroissement des capacités à lire et à écrire dans l'Angleterre des XI ${ }^{\mathrm{e}}-\mathrm{XIV}^{\mathrm{e}}$ siècles, a fait date ${ }^{6}$. Les années 1980 ont vu la parution d'autres contributions fondamentales ${ }^{7}$, sur un sujet dont l'historiographie est aujourd'hui en plein renouvellement, notamment dans le monde francophone : outre l'ouvrage déjà mentionné de Paul Bertrand, paru en 2015, il faut aussi citer le numéro de Médiévales coordonné en 2009 par Étienne Anheim et Pierre Chastang sur le thème des

2. J. W. Thompson, The Literacy of the Laity in the Middle Ages [Berkeley, 1939], New York, 1960,p. v : «Literacy during the Middle Ages may be measured almost wholly by the extent of the knowledge and use of the Latin language. »

3. P. Bertrand, Les Écritures ordinaires. Sociologies d'un temps de révolution documentaire (1250-1350), Paris, 2015, p. 13.

4. À partir de H. GrundmanN, «Litteratus - Illitteratus. Der Wandel einer Bildungsnorm vom Altertum zum Mittelalter », Archiv für Kulturgeschichte, 40 (1958), p. 1-65, article dans lequel l'historien allemand retrace l'évolution du concept médiéval de lettré depuis l'Antiquité tardive jusqu'à la fin du Moyen Âge, mettant ainsi en évidence la nécessité d'interpréter les termes de litteratus et d'illitteratus en fonction d'un contexte culturel variable.

5. M. Parkes, «The Literacy of the Laity », dans D. Daiches et A. Thorlby éd., Literature and Western Civilization, vol. II : The Medieval World, Londres, 1973, p. 555-577.

6. M. T. Clanchy, From Memory to Written Record. England, 1066-1307 [Londres, 1979], Oxford, 1993, p. 247 : "The increase in the number of persons who could read, at a minimal level, over the period 1066-1307 was thus a consequence of the demands of the "pragmatic" rather than the "cultivated" reader. »

7. Notamment F. H. BÄUML, « Varieties and Consequences of Medieval Literacy and Illiteracy », Speculum, 55/2 (1980), p. 237-265 ; В. Sтоск, The Implications of Literacy. Written Language and Models of Interpretation in the Eleventh and Twelfth Centuries, Princeton, 1983. Cf. infra n. 70. La collection «Utrecht Studies in Medieval Literacies » (Brepols) accueille depuis 1999 de nombreuses publications sur la littéracie médiévale. 
Pratiques de l'écrit ${ }^{8}$. L'idée de littéracie a ainsi renouvelé la compréhension du rapport des populations à la culture écrite, en dépassant un cadre épistémologique longtemps dominé par une équivalence implicite établie entre « écrit » et « livre », et donc entre «alphabétisation » et « capacité à lire des livres », soit des critères applicables en réalité à la seule culture savante, et valables pour certaines périodes seulement. L'influence des sciences sociales, et en particulier de l'anthropologie, a été décisive dans cette évolution ${ }^{9}$.

Les médiévistes envisagent désormais le fait d'être lettré comme une caractéristique sujette à maintes variations et nuances, selon les époques et les contextes. Les travaux d'Armando Petrucci ont montré qu'il pouvait exister, chez les moines copistes du haut Moyen Âge, un surprenant analphabétisme de lecture ${ }^{10}$ : en effet, les livres étaient alors surtout les vecteurs de la conservation d'un savoir qui était en priorité appris et transmis oralement, plutôt que lu. Cet usage conservatoire de l'écrit est aussi, à bien des égards, celui des chartes. Dans cette perspective, les $\mathrm{XI}^{\mathrm{e}}$-XIV ${ }^{\mathrm{e}}$ siècles apparaissent comme un moment clef de l'histoire culturelle européenne, un moment où la " révolution documentaire », maintes fois soulignée ${ }^{11}$, a profondément renouvelé les pratiques de la lecture et de l'écriture. Il semble en effet que la période voie la littéracie non seulement s'élargir, mais aussi se complexifier : on constate l'apparition et la diffusion de différentes façons d'aborder et de pratiquer la lecture et l'écriture, mettant en jeu les domaines respectifs du latin et des langues vernaculaires, ainsi que différentes utilisations de l'écrit, qui devient plus courant, mais aussi parfois aussi plus provisoire (notes, lettres) ${ }^{12}$ ou plus utilitaire (comptes, actes notariés), et peut-être plus personnel (mémoires, ricordi). On pouvait ainsi, aux $\mathrm{XIV}^{\mathrm{e}}-\mathrm{XV}^{\mathrm{e}}$ siècles, tel le marchand Francesco Datini, passer ses journées à lire et à écrire des lettres destinées à des correspondants

8. Médiévales, 56 (printemps 2009).

9. En particulier à partir de l'article de J. Goody et I. WATTS, « The Consequences of Literacy », Comparative Studies in Society and History, 5/3 (1963), p. 304-345. P. BERTRAND, Les Écritures ordinaires..., se réfère quant à lui explicitement aux travaux de Daniel Fabre, notamment D. FABRE éd., Écritures ordinaires, Paris, 1993.

10. A. PetruccI, «Lire au Moyen Âge », Mélanges de l'École française de Rome. Moyen $\hat{A}$ ge -Temps modernes, 96/2 (1984), p. 603-616 ; ID., Scrivere e leggere nell'Italia medievale, Milan, 2007.

11. Notamment par M. Clanchy, From Memory to Written Record... ; P. BerTRand, Les Écritures ordinaires...; P. CAMmARosano, Italia medievale. Struttura e geografia delle fonti scritte, Rome, 1991.

12. L'étude de la littéracie doit aussi tenir compte, dans la mesure du possible, de la problématique de la conservation des documents écrits. Les écrits "provisoires », pourtant essentiels à la juste évaluation du niveau de littéracie d'une société ou d'une catégorie sociale, manquent en général à l'appel, en particulier pour le haut Moyen Âge. On peut penser, par exemple, aux tablettes de cire. Voir sur ce sujet É. LaLou, «Les tablettes de cire médiévales », Bibliothèque de l'École des chartes, 147 (1989), p. 123-140. 
commerciaux disséminés dans plusieurs villes d'Europe, tout en n'étant pas, ou peu, capable d'aborder la littérature latine, et même vulgaire ${ }^{13}$. Le fait de posséder des livres ne signifie pas forcément qu'on les lisait ${ }^{14}$. L'évolution même de la forme de l'écrit (l'essor, par exemple, à la fin de notre période, de miscellanées "personnalisées ${ }^{15}$ ») révèle des approches individuelles caractéristiques d'un monde où la littéracie échappe à toute définition univoque. Doit-on d'ailleurs considérer, comme le font certains sociologues, que les «écrits ordinaires » sont les marqueurs d'une pratique diffuse, bien que socialement non reconnue, de l'écriture, y compris chez les personnes souvent considérées d'emblée comme non lettrées, en particulier les femmes ${ }^{16}$ ?

\section{Littéracie et genre}

Les travaux que nous allons maintenant considérer de plus près associent l'idée de littéracie à un autre concept, lui aussi venu de l'historiographie anglo-américaine, celui du genre. Leurs auteurs interrogent le rapport des femmes au monde de l'écrit, traditionnellement considéré, en particulier pour le Moyen Âge, comme essentiellement masculin. Ils démontrent de différentes manières la proximité des femmes médiévales avec le monde de l'écrit, et même avec la connaissance du latin, en interrogeant les pratiques genrées de la lecture, les modes et les moments de l'écriture féminine (en particulier chez les religieuses, mais pas seulement), l'idée d'une « auteurité » (authorship) féminine. Ce faisant, ces travaux ne posent pas simplement la question d'une approche "genrée » de la littéracie, ils remettent aussi en question certains cadres épistémologiques de l'histoire culturelle du Moyen Âge. Pourquoi, et depuis quand, les femmes apparaissent-elles aux historiens de la littérature, mais aussi plus largement à tous ceux qui, de près ou de loin, s'intéressent à l'histoire de l'écrit, comme essentiellement étrangères à la culture lettrée, sauf exceptions - les auteures célèbres - dûment reconnues et étudiées comme telles ? La question du rapport des femmes à la culture est donc d'autant plus stratégique qu'elle permet de repenser dans une autre perspective le rapport, structurant dans l'historiographie, entre une culture savante ou cléricale et une culture considérée comme laïque, voir profane. Les femmes, en effet, brouillent les

13. $C f$. S. Brambilla, "Libro di Dio e dell'anima certamente". Francesco Datini fra spiritualità e commercio librario », dans A. MANFredi et C. M. Monti éd., L'antiche e le moderne carte. Studi in memoria di Giuseppe Billanovich, Rome, 2007, p. 189-246.

14. Voir sur ce thème C. BEC, Les Livres des Florentins, 1413-1608, Florence, 1984.

15. Sur l'usage des miscellanées au Moyen Âge, voir A. Petrucci, Scrivere e leggere..., en part. p. 30. Petrucci tend toutefois à minimiser une pratique que la scolastique ne fait pas disparaitre, même si elle en change quelque peu l'utilité.

16. B. LAHIRE, La Raison des plus faibles. Rapport au travail, écritures domestiques et lectures en milieux populaires, Lille, 1993. 
pistes : même religieuses, elles ne sont pas des clercs et se trouvent de fait au contact des deux mondes, ne rechignant pas à utiliser concomitamment le latin et les langues vernaculaires. Beaucoup d'entre elles semblent en outre privilégier la lecture aux dépens de l'écriture, renforçant ainsi les spécificités de la littéracie du bas Moyen Âge, qui diffère profondément de la nôtre, mais aussi, peut-être, de celle des siècles précédents. L'étude d'une « littéracie genrée » ouvre ainsi des perspectives qui ne sont pas seulement liées à l'histoire des femmes et du genre, et s'inscrit directement dans le prolongement des évolutions de l'histoire culturelle médiévale précédemment décrites.

L'idée d'un rapport particulier des femmes à l'écrit s'appuie sur des travaux dont il importe ici de souligner l'évolution. Peter Dronke est l'un des premiers à s'être intéressé à la question dans son ouvrage Women Writers in the Middle Ages, publié en $1984{ }^{17}$. Malgré une démarche pionnière et une analyse fine et savante des textes, la perspective qu'il adopte, s'appuyant implicitement sur l'idée classique d'une féminité intemporelle (l'auteur rapproche les écrits de la martyre romaine Perpétue de ceux de Marguerite Porète ${ }^{18}$ ), lui interdit de penser précisément le rapport entre le genre (par définition socialement déterminé) et les pratiques de l'écriture et de la lecture. Peter Dronke écrit avant la diffusion en Europe du concept américain du gender $^{19}$. Son ouvrage pose néanmoins une question fondamentale : au cœur du rapport entre les femmes et l'écriture savante, on trouve la question de l'auctoritas, aussi bien au sens médiéval du terme (aucune femme n'est une autorité pour les écoles et les universités médiévales), qu'au sens moderne «d'auteurité »: la pleine responsabilité d'un texte médiéval est, encore aujourd'hui, difficilement attribuée à une femme ${ }^{20}$.

L'histoire des femmes lectrices, mécènes et dédicataires d'œuvres, propriétaires et utilisatrices de bibliothèques, c'est-à-dire l'histoire du rapport des femmes au livre, a suscité récemment de nombreuses et riches études. Nous citerons notamment le programme dirigé par Anne-Marie Legaré à partir de 2002, en collaboration avec Cynthia Brown à partir de

17. P. Dronke, Women Writers of the Middle Ages. A Critical Study of Texts from Perpetua $(† 203)$ to Maguerite Porete $(† 1310)$, Cambridge, 1984.

18. L'introduction illustre cette contradiction interne, car si l'auteur y précise qu'il ne cherche pas une féminité littéraire idéale (au sens platonicien du terme), il ajoute ensuite : « [Women] look at themselves more concretely and more searchingly than many of the highly accomplished men writers who were contemporaries », ibid., p. x. On trouve aussi, en fin de volume, des extraits des registres de Jacques Fournier (témoignages de femmes hérétiques), où les femmes manifesteraient une " self-consciousness » supérieure à celle des hommes.

19. En particulier à partir de la publication de J. Scotт, « Gender : A Useful Category of Historical Analysis », American Historical Review, 91/5 (1986), p. 1053-1075.

20. Sur ce point, $c f$. infra, « Le monopole du clericus litteratus ». 
2009, qui a donné lieu à la parution de deux volumes ${ }^{21}$. En 2007, le livre de D. H. Green, Women Readers in the Middle Ages, a repensé les nombreux acquis de l'histoire de la lecture et de la culture dans une perspective d'histoire du genre. Prenant comme point de départ les analyses de Herbert Grundmann ${ }^{22}$, D. H. Green met en évidence les limites du modèle du clericus litteratus. L'histoire du rapport des femmes à la lecture est ainsi l'occasion de redéfinir les limites, en réalité très perméables, entre les cultures savante et laïque, et ce d'autant plus que l'auteur définit ici autant les « lectrices» que les «lectures », c'est-à-dire les manières de lire ou d'aborder un texte écrit et/ou imagé, de l'assimiler, voire de le composer mentalement ${ }^{23}$.

\section{La littéracie au couvent}

D. H. Green est un spécialiste de la littérature germanique, comme tant d'autres qui ont écrit, et continuent d'écrire actuellement, sur la littéracie féminine. La fascination pour la mystique médiévale, et plus largement pour la littérature religieuse germanique, a joué un grand rôle dans le développement de ce champ d'étude. Les études de Peter Dinzelbacher sur la mystique féminine et, une fois encore, les interprétations fondatrices de Herbert Grundmann qui, dans son célèbre essai Religiöse Bewegungen im Mittelalter, a mis en relation l'émergence de la mystique allemande avec celle de la littérature vernaculaire, doivent être ici rappelées ${ }^{24}$. À partir des années 1990, la multiplication des études portant sur les relations entre les religieuses (en particulier les religieuses professant une règle, c'est-àdire les moniales) et le monde de l'écrit, a mis en évidence les capacités de ces femmes à copier, composer, enluminer, mais aussi à rassembler de grandes bibliothèques dans certains de leurs couvents, à l'opposé de l'image traditionnelle de nonnes peu cultivées, voire exclues de la haute

21. A.-M. Legaré éd., Livres et lectures de femmes en Europe entre Moyen Âge et Renaissance, Turnhout, 2007 ; A.-M. Legaré et C. J. Brown éd., Les Femmes, la culture et les arts en Europe entre Moyen Âge et Renaissance, Turnhout, 2016.

22. H. Grundmann, «Litteratus - Illitteratus... »; D. H. Green, Women Readers in the Middle Ages, Cambridge, 2007, p. 4.

23. D. H. Green (ibid., p. 23-27) propose ainsi une différence entre auctor et scriptor.

24. H. GRundmann, Religiöse Bewegungen im Mittelalter. Untersuchungen über die geschichtlichen Zusammenhänge zwischen der Ketzerei, den Bettelorden und der religiösen Frauenbewegungen im 12. und 13. Jahrhundert, und über die geschichtlichen Grundlagen der Deutschen Mystik, Berlin, 1935 ; trad. angl. S. Rowan, Religious Movements in the Middle Ages. The Historical Links between Heresy, the Mendicant Orders, and the Women's Religious Movement in the Twelfth and Thirteenth Century, with the Historical Foundations of German Mysticism, Notre Dame (IN), 1995 ; P. Dinzelbacher et D. Bauer éd., Frauenmystik im Mittelalter, Ostfildern, 1985 ; P. Dinzelbacher éd., Mittelalterliche Frauenmystik, Paderborn, 1993. On pourra se référer aussi à G. EPINEY-BuRgard et É. zum Brunn, Femmes troubadours de Dieu, Turnhout, 1988. 
culture par leur ignorance du latin. Les études de Gertrud Jaron Lewis ${ }^{25}$, Anne Winston-Allen ${ }^{26}$ et Jeffrey Hamburger ${ }^{27}$, mais aussi de Marie-Luise Ehrenschwendtner et Eva Schlotheuber ${ }^{28}$, ont ainsi fait resurgir l'écrit et l'écriture des moniales, aux confins entre la culture cléricale, qu'elles n'ignoraient pas mais qui n'était pas pleinement la leur, et une culture originale, proche de celle des laïcs, conjuguant de manière variable selon les temps et les lieux latin et langue vulgaire, pères de l'Église et mystiques contemporains.

Les communautés monastiques constituent un terrain d'études fécond pour le thème de la littéracie féminine, tout d'abord parce que les moniales, par le biais de la liturgie, avaient un rapport obligatoire à l'écrit ; ensuite parce qu'elles ont laissé des archives, des manuscrits, voire des catalogues de bibliothèques, qui constituent des ensembles documentaires cohérents. De tels ensembles sont difficilement repérables pour les femmes restées dans le monde, y compris les béguines, sauf dans le cas de certaines bibliothèques privées. Des projets internationaux portant sur l'étude de la littéracie des moniales médiévales ont récemment vu le jour et donné lieu à des publications riches et importantes. Le projet Nuns' Literacies in Medieval Europe, dirigé par Virginia Blanton, Veronica O'Mara et Patricia Stoop ${ }^{29}$, s'est donné pour ambition d'enquêter sur « le rapport entre les religieuses et les livres, qu'elles en aient été les auteures et/ou les lectrices, les commanditaires et/ ou les propriétaires ${ }^{30} »$. L'espace pris en compte par ce projet n'est pas limité à l'aire germanique ; toutefois les coordinatrices ont explicitement restreint leur investigation à l'Europe du Nord ${ }^{31}$. Deux volumes, regroupant

25. G. JARON LEWIS, By Women, for Women, about Women. The Sister-Books of Fourteenth Century Germany, Toronto, 1996.

26. A. Winston-Allen, Convent Chronicles. Women Writing about Women and Reform in the Middle Ages, University Park (PA), 2004.

27. Voir notamment J. HAmburger, The Visual and the Visionary. Art and Female Spirituality in Late Medieval Germany, New York, 1998.

28. M.-L. EhrenschwendTner, Die Bildung der Dominikarinnen in Süddeutschland, vom 13. bis 15. Jahrhundert, Stuttgart, 2004 ; E. SCHLOTHEuber, Klostereintritt und Bildung. Die Lebenswelt der Nonnen im späten Mittelalter, Tübingen, 2004.

29. V. Blanton, V. O’Mara, P. Stoop éd., Nuns' Literacies in Medieval Europe, vol. I : The Hull Dialogue, Turnhout, 2013 ; vol. II : The Kansas City Dialogue, Turnhout, 2015 [désormais désignés par les titres des deux tomes] ; vol. III, The Antwerp Dialogue, Turnhout, 2018. Ce troisième volume a été publié alors que j'avais déjà achevé la publication de ce texte.

30. The Hull Dialogue, p. Xv : «We seek to develop an understanding of how women religious across Europe interacted with books, as writers, readers, patrons, owners, benefactors, or inheritors of books ». J'ai choisi de simplifier la phrase dans sa traduction française, pour ne pas alourdir le texte.

31. The Hull Dialogue, p. XvI. On trouve toutefois deux articles sur l'Italie dans The Kansas City Dialogue : A. Ambrosio, « Literacy in Neapolitan Women's Convents : An Example of Female Handwriting in a Late Fifteenth-Century Accounts Ledger », p. 89-108 ; L. VANDI, «The Visual Vernacular : The Construction of Communal Literacy at the Convent 
les actes des deux premières rencontres, ont d'ores et déjà été publiés. On peut y lire de nombreuses contributions qui sont pour la plupart des études de cas (monastères particuliers, manuscrits ou œuvres spécifiques, études régionales). Bien que l'ensemble puisse parfois paraître un peu hétéroclite, ces volumes, qui rassemblent les recherches les plus récentes sur le sujet, font indéniablement émerger des perspectives nouvelles.

L'ouvrage d'Eva Schlotheuber, Jeffrey Hamburger, Margot Fassler et Susan Marti, intitulé Liturgical Life and Latin Learning at Paradies bei Soest, 1300-1425, a lui aussi pour objet d'étude la littéracie des moniales médiévales ${ }^{32}$. Il s'agit cette fois de l'étude minutieuse, voire exhaustive, des manuscrits liturgiques composés et enluminés au XIv ${ }^{\mathrm{e}}$ siècle par les moniales d'un seul monastère, celui du Paradies bei Soest, fondé en Westphalie au milieu du XIII ${ }^{\mathrm{e}}$ siècle. Le propos apparemment restreint est en fait le prétexte à une réévaluation plus générale des rapports des moniales médiévales avec la culture écrite : "Les manuscrits du Paradies nous forcent à repenser non seulement l'histoire de la branche féminine de l'ordre [dominicain] en Europe du Nord, mais aussi, plus généralement, l'histoire du savoir [learning], de la mystique, de la littérature et de l'art féminins ${ }^{33}$. » À travers une approche conjuguant histoire, histoire de l'art et musicologie, les quatre chercheurs ont bâti, en deux volumes très richement illustrés et comptant plus de 1500 pages, un véritable monument dédié à la culture des religieuses allemandes de la fin du Moyen Âge.

Au fil des pages des manuscrits du monastère du Paradies, les références scripturaires et patristiques s'accumulent, qu'il s'agisse de citations écrites ou iconographiques. L'habileté des sœurs à manier l'Écriture sainte, à saisir et à réinterpréter le sens de la liturgie et même à livrer leurs propres interprétations théologiques, se manifeste en particulier dans l'élaboration d'illustrations complexes alliant, pour chaque fête, références à l'Ancien et au Nouveau Testament, le tout dans un souci constant, de la part des religieuses, de se situer à la fois par rapport à la divinité et par rapport au monde. On notera, en particulier, l'abondance des références à Marie et à Jean l'Évangéliste, dont la relation était assimilée par les moniales au lien qui les liait aux frères prêcheurs ${ }^{34}$. Les prières micrographiques, minuscules textes glissés entre les partitions, ou à l'intérieur des images, et qui n'étaient à destination que de celles qui pouvaient ou voulaient les lire (dans le sens où, leur module

of Santa Maria in Pontetetto », p. 171-190. On pourra aussi se reporter à L. Miglio, Governare l'alfabeto. Donne, scrittura e libri nel Medioevo, Rome, 2008.

32. M. Fassler, J. Hamburger, S. Marti, E. Schlotheuber, Liturgical Life and Latin Learning at Paradies bei Soest, 1300-1425, Münster, 2016 [désormais Liturgical Life].

33. Liturgical Life, vol. I, p. 2 : « The books from Paradies compel a rethinking, not only of the history of the Order's female branch in northern Europe, but also more generally of female learning, mysticism, literature, and art ».

34. Liturgical Life, chap. 23. Voir aussi J. Hamburger, St John the Divine. The Deified Evangelist in Medieval Art and Theology, Berkeley, 2002. 
d'écriture étant minuscule par rapport à celui des textes liturgiques, il fallait s'approcher du livre pour les découvrir), sont la manifestation de la profusion et de la profondeur du savoir et de la culture de ces moniales.

L'interrogation première porte donc sur la formation des sœurs : où, et comment, ont-elles acquis leur savoir? L'hypothèse d'une spécificité de la politique de l'ordre dominicain envers les femmes est évoquée, notamment à travers les décisions d'Humbert de Romans, maître général de l'ordre de 1254 à 1263, d'Hermann de Minden, provincial de Teutonie entre 1286 et 1294, mais aussi de Maître Eckhart, premier provincial (1303-1311) de la nouvelle province de Saxe à laquelle appartenait le monastère du Paradies ${ }^{35}$. D'autres études ont montré, de fait, que les monastères dominicains de l'Empire germanique ont été des centres de culture religieuse de premier plan : Unterlinden de Colmar ${ }^{36}$, Sainte-Catherine de Nuremberg, SainteCatherine à Saint-Gall ${ }^{37}$. La richesse des bibliothèques de communautés religieuses non dominicaines (telles celle des cisterciennes de Kirchheim) a cependant été soulignée dans d'autres études ${ }^{38}$. Plusieurs contributions des volumes du projet Nuns' Literacies reviennent sur cet aspect. Anne Winston-Allen y publie une synthèse sur l'aire géographique dont elle est familière, c'est-à-dire celle des régions rhénanes de la Suisse jusqu'aux Pays-Bas actuels, où elle recense 140 manuscrits connus pour avoir été composés par des religieuses ${ }^{39}$.

La plupart de ces travaux rappellent que les novices, qui devaient être capables de lire et de chanter l'office, suivaient à l'intérieur de leurs communautés un cursus de formation plus ou moins développé. Toutefois, il apparaît évident que les niveaux de littéracie variaient d'une communauté à une autre et, à l'intérieur de ces communautés, d'une moniale à l'autre. Toutes les moniales en effet ne savaient pas écrire, toutes ne savaient pas

35. Voir en particulier Liturgical Life, vol. I, p. 69-70 (commentaire du sermon de Maître Eckhart sur Marthe et Marie). 2000.

36. J. Hamburger, M. Blondel, C. Leroy éd., Les Dominicaines d'Unterlinden, Colmar,

37. S. Mengis, Schreibende Frauen um 1500 : Scriptorium und Bibliothek des Dominikanerinnenklosters St. Katharina St. Gallen, Berlin, 2013.

38. A. Sснвомм, Die Bibliothek des ehemaligen Zisterzienserinnenklosters Kirchheim am Ries : Buchpflege und geistiges Leben in einem schwäbischen Frauenstift, Tübingen, 1998. Dans l'aire italienne, les dominicaines ne semblent pas avoir possédé des bibliothèques comparables à celles de leurs consœurs allemandes : $c f$. S. DuvaL, " Usages du livre et de l'écrit chez les moniales dominicaines observantes, Italie, première moitié du $\mathrm{xv}^{\mathrm{e}}$ siècle », dans N. Bériou, M. Morard, D. Nebbiat éd., Entre stabilité et itinérance. Livres et culture et des ordres mendiants $X I I I^{-}-X V^{e}$ siècle, Turnhout, 2014, p. 215-228. Une étude détaillée a été consacrée aux manuscrits des brigittines : R. MiRIELLo, I manoscritti del monastero del Paradiso a Firenze, Florence, 2007.

39. A. Winston-Allen, "Outside the Mainstream : Women as Readers, Scribes and Illustrators of Books in Convents of the German-Speaking Regions », The Kansas City Dialogue, p. 191-207. On pourra aussi se reporter à ce site internet, de la même auteure : [en ligne] http://www.agfem-art.com/ [consulté le 21 septembre 2018]. 
le latin. Quant aux moniales les plus cultivées, elles apparaissent mues par un goût de l'étude qui n'était évidemment pas partagé par toutes. Beaucoup d'entre elles, par ailleurs, possédaient des compétences qui étaient utiles à la vie de la communauté, mais qui n'étaient pas forcément requises pour accéder au statut de moniale de chœur (tenir les comptes, enluminer les manuscrits, connaître la musique...). Ces éléments, qui nous montrent les communautés de moniales comme une somme d'individualités bien plus que comme des groupes homogènes, ne sont pas surprenants : rien de très différent, au fond, de ce que l'on sait des communautés d'hommes - sauf dans le cas des ordres explicitement voués à l'étude, tels les frères prêcheurs. La question qui émerge de ce constat, dès lors, n'est pas de savoir si les religieuses étaient instruites - elles l'étaient forcément, en moyenne, plus que les femmes restées dans le siècle -, mais plutôt en quoi leur instruction pouvait être « genrée », c'est-à-dire différente de celles des hommes.

\section{Le latin a-t-il un genre?}

L'apprentissage du latin apparaît comme un thème presque emblématique des études sur la littéracie des religieuses. Nous entrons ici dans un domaine plus polémique, qui touche à la définition d'une culture " savante », dont la caractéristique serait l'usage du latin. Cet aspect de la thématique qui nous occupe ne met pas en jeu seulement la différence entre hommes et femmes, mais aussi, et peut-être surtout, le contraste entre clercs et laïcs. Maîtriser le latin, c'est en effet pouvoir aborder la culture cléricale et tout particulièrement, à la fin du Moyen Âge, celle des universités. On a longtemps considéré d'emblée que les femmes, moniales ou non, n'y avaient pas accès. Leurs écrits, le plus souvent caractérisés à la fin du Moyen Âge par l'usage des langues vernaculaires, étaient par conséquent perçus comme la manifestation d'une culture secondaire ou du moins - selon le point de vue adopté - alternative. Les choses ne sont évidemment pas aussi simples, et les limites entre ces différents domaines culturels, comme entre l'emploi $\mathrm{du}$ latin et des langues vernaculaires, sont bien moins rigides que ce que l'on a pu croire. Or, les études sur les moniales contribuent mieux que d'autres à démontrer toute la complexité de ce sujet.

Dans quelle mesure les religieuses savaient-elles le latin ? Ne récitaientelles pas, pour certaines, l'office par cœur, faute de savoir réellement lire ? Ces questions ont souvent été posées ; Monica Hedlund les aborde avec méthode dans une riche contribution consacrée au monastère suédois de Vadstena $^{40}$. Pour Eva Schlotheuber ${ }^{41}$, il y a dans ce domaine, au XIV ${ }^{\mathrm{e}}$ siècle, une spécificité des régions septentrionales de l'Allemagne, où aurait perduré

40. M. HedLund, « Nuns and Latin, with Special Reference to the Birgittines of Vadstena », The Hull Dialogue, p. 97-118.

41. Liturgical Life, chap. 3. Voir aussi E. SCHLOtheuber, Klostereintritt und Bildung... 
plus longtemps un enseignement du latin typique du Moyen Âge central. Les moniales du Paradies bei Soest, comme leurs consœurs du second monastère saxon de moniales dominicaines, à Lemgo, disposaient d'une bibliothèque qui contenait aussi bien des ouvrages en langue vernaculaire qu'en langue latine - une caractéristique que l'on retrouve à Colmar et à Nuremberg. Les ouvrages latins de ces bibliothèques étaient-ils destinés aux seuls chapelains et directeurs spirituels des moniales ? Les livres liturgiques du Paradies bei Soest démontrent que les moniales mariaient aux enseignements qu'elles recevaient des clercs une approche toute personnelle de la culture religieuse, et même de la théologie. Tel est l'aspect qui apparaît à travers l'étude des complexes illustrations de ces manuscrits, mais aussi des séquences latines que les moniales composaient elles-mêmes pour leurs offices, comme le montrent les études musicologiques de Margot Fassler ${ }^{42}$. L'usage conjugué du latin et des langues vernaculaires est aussi rappelé par Cynthia Cyrus ${ }^{43}$, qui démontre l'existence, dans les écrits des moniales de Vienne (Autriche), d'un continuum entre latin et allemand. Quant à Patricia Stoop, elle nous montre, dans un très bel article sur les religieuses du monastère de Jéricho à Bruxelles, que les moniales pouvaient écrire un texte en langue vulgaire tout en y insérant des citations latines ${ }^{44}$. Le cas des religieuses bruxelloises, qui organisent, autour de leur scrijfcamere, leur « chambre à écrire », un travail collectif de rédaction des sermons délivrés devant leur communauté, est, à bien des égards, exemplaire. Il apparaît en effet que, parmi les moniales bruxelloises, beaucoup savaient écrire, mais peu nombreuses étaient celles qui possédaient la maîtrise du latin, et moins encore celles qui étaient capables de retrouver, copier et référencer les citations scripturaires ou patristique : la transcription des sermons nécessitait donc une réunion de compétences qui ressemble peut-être à celle qui présida à la composition des manuscrits du Paradies bei Soest, et en particulier à celle du splendide graduel D1 $1^{45}$, manuscrit sur lequel les auteurs de Liturgical Life and Latin Learning s'attardent le plus, et qui est presque entièrement reproduit dans le deuxième volume. Ce travail en équipe se développe à l'intérieur de la clôture du monastère, dont le relatif isolement change nécessairement le rapport au savoir, aussi bien dans son acquisition que dans sa diffusion. Le travail des moniales de Bruxelles, sorte de reportationes savantes, processus que Patricia Stoop décrit aussi dans une seconde contribution pour le

42. Liturgical Life, chap. 9-11.

43. C. J. Cyrus, « Vernacular and Latinate Literacy in Viennese Women's Convents », The Hull Dialogue, p. 119-132

44. P. Stoop, «From Reading to Writing : the Multiple Levels of Literacy of the Sister Scribes in the Brussels Convent of Jericho », The Kansas City Dialogue, p. 47-66.

45. Aujourd'hui conservé à la Bibliothèque universitaire de Düsseldorf. 
monastère de cisterciennes de La Cambre (Ter Kameren, Ixelles) ${ }^{46}$, est à bien des égards typique de ce monde soumis à la stricte clôture, où le travail tient lieu de méditation commune et peut conduire, comme le démontre le graduel du Paradies bei Soest, à l'élaboration d'un savoir autoréférentiel qui cimente la communauté dans sa pratique liturgique quotidienne et dans la perception qu'elle a d'elle-même.

On connaît, par ailleurs, de nombreux cas de moniales « exceptionnelles », qui ont brillé par leur culture latine, comme Hildegarde de Bingen bien sûr ${ }^{47}$, mais aussi comme la prieure dominicaine de Colmar Elisabeth Kempf, dont la parfaite connaissance du latin fit, à la fin du $\mathrm{XV}^{\mathrm{e}}$ siècle, l'admiration de son maître général, qui put aisément discuter avec elle dans cette langue ${ }^{48}$. D'autres exemples pourraient être cités pour l'Italie de la fin du Moyen Âge ${ }^{49}$. À partir du XIV ${ }^{\mathrm{e}}$ siècle pourtant, il devient fréquent de traduire à l'intention des moniales les œuvres non liturgiques, qu'il s'agisse d'œuvres spirituelles (saint Augustin par exemple) ou normatives (les règles de vie des religieuses) ${ }^{50}$. Cette vague de traductions révèle-telle, ainsi que le soulignent plusieurs contributions, un abandon progressif du latin de la part des religieuses ? Il est ici tentant de faire du XIV siècle le moment d'une transition entre latin et langue vulgaire qui aurait lieu au sein des monastères en parallèle avec la multiplication, dans le monde laïc, des écrits vernaculaires ${ }^{51}$. L'idée d'un recul du latin dans les communautés de femmes tient toutefois pour acquis un élément qui ne l'est pas (la bonne

46. P. Stoop, « Nuns' Literacy in Sixteenth-Century Convent Sermons from the Cistercian Abbey of Ter Kameren », The Hull Dialogue, p. 293-312.

47. Sur Hildegarde et, plus généralement, la place de la littéracie féminine dans l'Église médiévale, on pourra se reporter à M. E. Góngora et T. de Hemptinne éd., The Voice of Silence. Women's Literacy in a Men's Church, Turnhout, 2004.

48. Voir J. AnCelet-Hustache, « Les "Vitae Sororum" d'Unterlinden. Édition critique du ms. 508 de la bibliothèque de Colmar », Archives d'histoire doctrinale et littéraire du Moyen $\hat{A} g e, 5$ (1931), p. 317-513 (en part. p. 504 sq.).

49. Telle la moniale vénitienne Elisabetta Tomasini qui, à la fin du XIV ${ }^{\mathrm{e}} \mathrm{s}$., était surnommée la teologa par Jean Dominici, tandis que ses consœurs la considéraient comme uno doctor. Voir S. Duval, « Usages du livre... », p. 218.

50. Elisabeth Kempf en particulier traduit des ouvrages pour ses sœurs qui ne savent pas le latin ( $c f$. n. 50). En Italie, le processus est ancien : au XIv ${ }^{\mathrm{e}}$ s., les dominicains du couvent de Pise, tel Domenico Cavalca, composent ou traduisent des œuvres en langue vulgaire pour les moniales et les femmes pieuses. Les règles de vie sont traduites elles aussi, afin que les moniales les comprennent : on retrouve ces prescriptions dès la fin du XIII ${ }^{\mathrm{e}} \mathrm{s}$., tant dans les ordinationes des maîtres généraux de Teutonie que dans ceux de la province romaine. Voir M. L. EhrenschwendTner, «Puellae Litteratae : the Use of the Vernacular in the Dominican Convents of Southern Germany », dans D. W AтT éd., Medieval Women in their Communities, 1997, p. 49-71 ; S. Duval, "Comme des anges sur terre ». Les moniales dominicaines et les débuts de la réforme observante, 1385-1461, Rome, 2015, en part. p. 589-596.

51. Sur la question de la diglossie médiévale, on pourra se reporter notamment à F. H. BäumL, « Varieties and Consequences... »; S. Lusignan, Parler vulgairement. Les intellectuels et la langue française aux XIII et XIV siècles, Montréal, 1986 ; A. GAUTIER, « La voix des laïcs? L'écrit vernaculaire dans l'Europe du Nord aux siècles centraux du Moyen 
connaissance du latin de la part de la majorité des religieuses durant la période précédant le $X^{\mathrm{e}}{ }^{\mathrm{e}}$ siècle) ; il convient donc d'être nuancé. Le fait qu'Elisabeth Kempf, citée plus haut, traduise un grand nombre de textes à l'intention de ses consœurs est peut-être l'effet d'un certain déclassement du latin dans le cursus de formation des religieuses, mais c'est aussi la conséquence d'une augmentation des exigences liées au contenu même de cette formation, dans le contexte, à Colmar, d'une réforme observante aussi stricte que brillante. Le renforcement des réglementations visant les communautés religieuses féminines précède d'ailleurs l'Observance ${ }^{52}$, et explique pour une bonne part que les autorités religieuses aient souhaité traduire les textes réglementaires. Rappelons en effet que le nombre de moniales (mais aussi de semi-religieuses : béguines, recluses, pénitentes...) a considérablement augmenté à partir de la fin du XII ${ }^{\mathrm{e}}$ siècle, pour atteindre, au début du XIV ${ }^{\mathrm{e}}$ siècle $^{53}$, des niveaux qui n'ont plus rien de comparable avec le nombre réduit des abbayes féminines du Moyen Âge central, dont le recrutement était notoirement élitiste. La diversification des modes de vie religieux, l'élargissement du recrutement aux classes plus modestes, la multiplication des monastères de femmes (clarisses, dominicaines, puis brigittines...), bouleversent le paysage religieux féminin entre les XII et $\mathrm{XV}^{\mathrm{e}}$ siècles. Ces phénomènes ont eu des conséquences non seulement sur la gestion des communautés féminines par l'institution ecclésiale, mais aussi sur le niveau et le contenu de l'éducation dispensée dans les cloîtres de moniales, et enfin sur le rôle social et culturel de ces communautés.

\section{Les moniales, des laïques comme les autres?}

La question de la littéracie des moniales doit, de fait, être comprise dans un contexte plus large que celui de la seule histoire de la littérature et du savoir religieux. On notera, à ce sujet, la très intéressante contribution de Thomas Mertens sur la prière en langue vernaculaire ${ }^{54}$, qui établit un lien décisif entre la littéracie des religieuses et semi-religieuses et les évolutions

Âge », dans B. BéthouART et L. DucERF éd., Les Laïcs dans les religions, Boulogne-sur-Mer, 2014 (Les Cahiers du Littoral, $2^{\mathrm{e}}$ série, 13), p. 13-23.

52. La stricte clôture est rendue obligatoire pour toutes les moniales par la décrétale Periculoso en 1298. Les communautés rattachées aux ordres mendiants (clarisses, dominicaines) doivent aussi suivre des règles de vie très austères sur ce point, et dont le respect littéral est promu par les réformateurs observants aux Xve s. Voir S. Duval, "Comme des anges sur terre »..., chap. 1, II.

53. Les monastères de dominicaines de l'aire germanique sont particulièrement nombreux, puisque Bernard Gui en dénombre 58 à la fin du XIII ${ }^{\mathrm{s}} \mathrm{s}$. (province de Teutonie), et Antonin de Florence 70 en 1450 (provinces de Teutonie et de Saxe). Cf. S. Duval, "Comme des anges sur terre »..., p. 43.

54. T. Mertens, "Praying in the Vernacular : Middle Dutch Imitative Forms of the Divine Office from the 1370s to 1520s », The Hull Dialogue, p. 133-148. 
de leurs formes de vie, mais aussi, plus généralement, des pratiques de piété (rôle de la devotio moderna) : une remise en contexte qui ouvre la voie à des interprétations plus larges de la littéracie féminine. Il s'agit d'un petit écart par rapport à la définition initiale du projet Nuns' Literacies, qui devait a priori prendre en compte les seules moniales ; cet écart est toutefois pleinement justifié, et peut-être même salutaire, dans la mesure où moniales professes et semi-religieuses étaient, en particulier dans les villes de la fin du Moyen Âge, en relation constante. Il n'était pas rare en effet que des communautés plus informelles (béguines, recluses) adoptent officiellement une règle, devenant ainsi des communautés de moniales professes. Les religieuses étaient en outre, même cloîtrées, en contact avec leurs familles, leurs partenaires économiques, leurs patrons laïcs, ou encore les simples fidèles qui venaient à elles. On notera, à ce propos, l'absence de contributions portant sur la présence de jeunes filles laïques (c'est-à-dire non engagées dans la vie religieuse) au sein des monastères où elles recevaient une éducation : leur existence est pourtant bien attestée, notamment pour l'Italie de la fin du Moyen Âge ${ }^{55}$.

Quel est, d'ailleurs, l'impact de la focalisation de ces projets sur l'Europe du Nord ? On peut en effet s'interroger sur les conséquences de cette restriction quant à la typologie des sources envisagées, et particulièrement sur l'absence de prise en compte des documents de la pratique, beaucoup plus présents (mais aussi, peut-être, plus étudiés) dans les corpus de sources du sud de l'Europe. Symétriquement, on pourra s'étonner de l'absence de projets de recherches d'envergure sur ce sujet en Italie, en France et en Espagne ${ }^{56}$, qui empêche, du moins pour le moment, l'établissement d'une vraie comparaison ${ }^{57}$. Les registres de gestion des monastères, les écritures comptables, fort nombreux en Italie ${ }^{58}$, mais aussi les chartes, parfois écrites en Allemagne par les moniales elles-mêmes ${ }^{59}$, démontrent en effet que les religieuses détenaient aussi une culture écrite

55. Les educande, jeunes filles éduquées au monastère avant d'être mariées, sont nombreuses déjà au $\mathrm{Xv}^{\mathrm{e}} \mathrm{s}$.; elles y apprennent notamment à lire et à écrire. $C f$. S. DuvaL, "Scrivere, contare, gestire. I libri di amministrazione dei monasteri femminili fiorentini (1320-1460) » dans C. Bianca et A. Scattigno, éd., Scritture, carismi, istituzioni. Percorsi di vita religiosa in età moderna. Studi per Gabriella Zarri, Rome, 2018, p. 85-104.

56. Bien que l'histoire moderne ait été, en revanche, particulièrement féconde en ce domaine. On pourra se référer à T PLEBANI, Il « genere » dei libri. Storie e rappresentazioni della lettura al femminile e al maschile tra Medioevo e età moderna, Milan, 2003 ; X. voN TIPPELSKRICH, Sotto controllo. Letture femminili in Italia nella prima età moderna, Rome, 2011. Pour l'Espagne, notamment, I. Poutrin, Le Voile et la plume. Autobiographie et sainteté féminine dans l'Espagne moderne, Madrid, 1995.

57. Cf. supra n. 31. Il faut signaler la parution imminente d'un recueil d'autographes féminins médiévaux : G. Murano éd., Autographa II. 1. Donne, sante e madonne (da Matilde di Canossa ad Artemisia Gentileschi), Imola, 2018.

58. S. Duval, « Scrivere, contare, gestire...».

59. Liturgical Life, vol. I, p. 78. 
« pratique » remarquable, qui leur permettait de gérer leur communauté et leurs domaines. L'analyse de ce type de sources, qui révèlent la familiarité quotidienne des femmes avec le monde de l'écrit, permettrait peut-être aussi d'échapper à la surreprésentation de la littérature religieuse (et de l'écrit religieux en général) que l'on constate dans les études actuelles. Si le thème de la connaissance du latin pose la question de l'accès des religieuses à la culture cléricale, la capacité de certaines religieuses à tenir des comptes complexes pose celle de la pénétration de la culture laïque (ici, plus spécifiquement, de la culture marchande) à l'intérieur des cloîtres . Au-delà de ces questions, c'est bien le problème de l'éducation des filles au Moyen Âge qui est ici posé. Qu'apprennent les novices au monastère ? Qu'apprennent les petites filles avant d'entrer en religion ? Que penser, à ce sujet, des recommandations d'Humbert de Romans, qui incite les parents à instruire les filles qu'ils destinent au cloître ${ }^{60}$ ?

La question de l'instruction des femmes au Moyen Âge reste un vaste domaine d'enquête. Les interprétations de D. H. Green méritent à ce sujet d'être citées : il avance en effet que, dans l'aristocratie médiévale, les femmes, habituées à lire et à méditer sur leurs ouvrages pieux, étaient tout simplement plus lettrées que leurs maris. En effet, dit-il, ceux-ci devaient apprendre à combattre, tandis que leurs épouses, destinées à demeurer le plus souvent entre les murs du foyer, pouvaient s'adonner paisiblement à la lecture - une activité d'autant mieux perçue qu'elle était souvent liée à une pratique personnelle de la piété religieuse. On pense ici au célèbre Manuel de Dhuoda, qui témoigne aussi bien de la préoccupation de cette aristocrate $\mathrm{du} \mathrm{IX}^{\mathrm{e}}$ siècle pour ses enfants que de sa méditation quotidienne des textes sacrés en son domaine d'Uzès ${ }^{61}$. Cette méditation est aussi celle des riches maîtresses de maison du $\mathrm{XV}^{\mathrm{e}}$ siècle qui, à l'image des moniales, souhaitent posséder un livre d'heures et à qui les directeurs de conscience prescrivent une lecture assidue du bréviaire, parfois même préférée à la fréquentation des églises, considérée comme potentiellement périlleuse parce qu'elle entraîne une sortie récurrente des murs protecteurs du foyer domestique ${ }^{62}$.

\section{Faut-il étudier la littéracie médiévale dans une perspective genrée?}

Méditation et éducation : ces deux usages du livre et de l'écrit apparaissent comme consubstantiels à une certaine définition de la maternité médiévale. Le bréviaire ou le livre d'heures sont en effet bien souvent le support

60. Ibid., p. 71.

61. Dhuoda, Manuel pour mon fils, éd. P. Riché, Paris, 1997.

62. G. HASENOHR, « La vie quotidienne de la femme vue par l'Église. L'enseignement des "journées chrétiennes" à la fin du Moyen Âge », dans H. ApPELT éd., Frau und Spätmittelalterlicher Alltag, Vienne, 1986, p. 19-102. 
d'une première instruction des petits enfants qui marie indissociablement l'apprentissage de la lecture (mais non de l'écriture ${ }^{63}$ ) à celui de la méditation $^{64}$, ainsi que le rappellent les représentations, courantes à la fin du Moyen Âge, de la Madone ou de sainte Anne tenant un livre devant leur enfant. $\mathrm{Au}$ début du $\mathrm{Xv}^{\mathrm{e}}$ siècle, Jean Dominici, dominicain rigoriste et opposé aux humanistes ${ }^{65}$, dédie à sa fille spirituelle Bartolomea degli Alberti un traité sur la vie familiale, dans lequel il dit tout son rejet de la «nouvelle » éducation que l'on donne alors aux garçons à Florence. Par réaction contre cette nouveauté, c'est bien à la mère (Bartolomea est une femme instruite) qu'il demande expressément de superviser l'éducation de ses fils ${ }^{66}:$ il se réfère ici à un usage bien ancré dans la société médiévale. Le livre, ou plutôt certains livres - les livres pieux en particulier - peuvent donc, aussi, être typiquement féminins. Les bréviaires comme les «petits livres de femmes », libricini da donna, sont des éléments récurrents des trousseaux des jeunes épouses italiennes des XIV ${ }^{\mathrm{e}}-\mathrm{XV}^{\mathrm{e}}$ siècles ${ }^{67}$; on retrouve d'ailleurs des libricini parmi les productions des moniales florentines : il fait peu de doute qu'il s'agissait de petits ouvrages pieux destinés aux femmes ${ }^{68}$.

Pour autant, la littéracie féminine ne se résume pas à ces éléments, tout comme la lecture pieuse n'est pas un exercice purement féminin. Les travaux développés actuellement par Sabrina Corbellini, Margriet Hoogvliet et Bart Ramakers à l'Université de Groningue le démontrent. S'intéressant aux pratiques de la lecture religieuse en Europe entre Moyen Âge et époque moderne ${ }^{69}$, dans une perspective qui reste marquée à la fois par l'histoire de la Réforme et par les études sur la littéracie des

63. Ce qui correspond, par exemple, aux prescriptions du chevalier de La Tour Landry. Cf. D. H. Green, Women Readers..., p. 86-87.

64. J. Delumeau éd., La Religion de ma mère. Le rôle des femmes dans la transmission de la foi, Paris, 1992 ; C. KLAPISCH-ZUBER, « Les clefs florentines de Barbe Bleue. L'apprentissage de la lecture », dans EAD., La Maison et le Nom. Stratégies et rituels dans l'Italie de la Renaissance, Paris, 1990, p. 309-330.

65. Jean Dominici, Lucula Noctis, éd. R. Coulon, Paris, 1908. Voir aussi C. Mésionat, Poetica Theologia : La "Lucula Noctis" di Giovanni Dominici e le dispute letterarie tra '300 $e$ '400, Rome, 1984. Jean Dominici expose le même point de vue dans le traité du Governo familiare ( $c f$. note suivante).

66. Jean Dominici, Regola del governo di cura familiare, éd. D. SALVI, Florence, 1860, p. 133-136.

67. C. KLAPISCH-ZuBER, «Les clefs florentines »

68. S. Duval, « Usages du livre... ».

69. S. Corbellini, M. Hoogvliet, B. Ramakers éd., Discovering the Riches of the Word: Religious Reading in Late Medieval and Early Modern Europe, Leyde, 2015 ; S. CoRBELLINI éd., Cultures of Religious Reading in the Late Middle Ages : Instructing the Soul, Feeding the Spirit, and Awakening the Passion, Turnhout, 2013. Voir aussi M. HoogvLIET, " "Une petite instruction pour femme séculière" : lectures religieuses des femmes et bibliothèques à la fin du Moyen Âge », La Revue de la BNU (Bibliothèque nationale universitaire de Strasbourg), 14 (2016), p. 36-43. 
laïcs ${ }^{70}$, ils mettent en évidence la très grande diffusion, en particulier dans les milieux urbains, d'ouvrages pieux à destination des laïcs, hommes et femmes. L'un des apports majeurs de ces études est peut-être la description souvent minutieuse de pratiques de lecture qui engagent les lecteurs à une « participation active ${ }^{71}$ » et qui se déroulent en un temps qui n'est pas celui du simple déchiffrement des caractères, mais bien celui du cheminement spirituel. La lecture suit ainsi le rythme de la méditation, et non l'inverse, le stade ultime de la lecture-méditation étant la contemplation ${ }^{72}$. On retrouve cette idée dans les travaux qui remettent aujourd'hui en valeur le rôle de la liturgie dans les communautés religieuses féminines, en particulier dans le cadre de la réforme observante ${ }^{73}$. Le soin infini apporté par les moniales du Paradies bei Soest à la création de leurs livres liturgiques doit bien sûr être compris ainsi : ils n'étaient pas les simples supports de la lecture, ni même du chant, mais bien des supports de l'accès au divin. Leur contenu était d'ailleurs, tout comme les livres pieux destinés aux laïcs, « multimodal » : les moniales y découvraient à la fois des prières destinées à être lues individuellement (prières micrographiques), les textes destinés à être chantés collectivement, et enfin des images aux significations complexes destinées à favoriser la méditation.

Ce que les études actuelles sur la littéracie médiévales mettent donc en évidence, c'est peut-être avant tout le foisonnement des pratiques et des supports de l'écriture, et surtout de la lecture, qui déborde les schémas classiques de l'histoire culturelle et remet profondément en question les limites auxquelles on a encore tendance à se référer. Parmi ces schémas - mais ce n'est peut-être au fond qu'un aspect parmi d'autres - celui d'une culture principalement masculine ne tient plus. Les femmes médiévales avaient accès aux lettres, et parfois, dans certains milieux comme l'aristocratie, peut-être même plus que les hommes. Par conséquent, bien plus qu'un éventuel «genre » de la littéracie, ce que les études actuelles font apparaître, comme par contre-jour, c'est la formation progressive des catégorisations culturelles dont nous sommes les héritiers et, en particulier, la hiérarchisation des acteurs de la culture écrite, au sommet de laquelle on trouve les clercs.

70. En particulier par le concept de textual communities proposé par B. STоcк, The Implications of Literacy...

71. «Active participation»: S. CoRbELLINI : «Uncovering the Presence : Religious Literacies in Late Medieval Italy » dans EAD. et al., Discovering the Riches..., p. 68-87.

72. S. Corbellini rejoint ici M. Clanchy sur l'analyse de cette forme particulière de lecture : $c f$. M. Clanchy, From Memory to Written Record..., p. 195.

73. Voir sur ce point C. TAYLOR Jones, Ruling the Spirit. Women, Liturgy and Dominican Reform in Late Medieval Germany, Philadelphie, 2018. 


\section{Le monopole du clericus litteratus (et sa postérité)}

C'est dans cette perspective qu'Eva Schlotheuber s'interroge sur la perception que les religieuses de la fin du Moyen Âge, dont beaucoup étaient très instruites, avaient d'elles-mêmes et que les autres avaient d'elles ${ }^{74}$. Tandis que les clercs suivaient (à partir de la deuxième moitié du XIII ${ }^{\mathrm{e}}$ siècle) un cursus universitaire, qu'ils étaient reconnus, grâce aux efforts menés par l'Église depuis la réforme grégorienne, comme les détenteurs d'une autorité spirituelle devenue une sorte de monopole culturel, les moniales demeurèrent fidèles à une vie de prière et de méditation (qui était aussi celle des moines), renforcée par une insistance renouvelée sur leur statut de Sponsae Christi. Il faudrait ainsi comprendre l'image, récurrente à la fin du Moyen Âge dans l'art religieux, de la moniale ou de la sainte tenant un livre, comme étant moins le symbole de «l'apprentissage des lettres [par les religieuses] que celui de leur méditation et de la conversion totale de leur esprit vers le divin ${ }^{75} \gg$. En revanche, la représentation d'hommes tenant leurs propres œuvres sous forme de livres serait bien la manifestation d'une autorité intellectuelle ${ }^{76}$. Cette perception différenciée ne signifie donc pas que les moniales n'étaient pas lettrées, voire, pour certaines, les détentrices d'un savoir intellectuel et théologique raffiné, mais plutôt que ce savoir « féminin » ne bénéficiait pas (ou plus) de la même reconnaissance que celui des hommes. L'avènement de l'université, lieu de savoir exclusivement masculin et clérical, est sans nul doute un facteur déterminant dans cette évolution. La mise en valeur d'un savoir analytique masculin a renvoyé peu à peu, par contraste (la diffusion des idées aristotéliciennes aidant ${ }^{77}$ ), le « savoir féminin » à la sphère émotionnelle et corporelle. Cet état de fait n'empêcha pas les femmes de continuer à développer une culture religieuse originale et même assez largement reconnue. Cependant, les discours religieux féminins, qui bénéficiaient d'une large audience, étaient désormais assimilés à une science infuse d'origine divine dont les femmes n'auraient été que des réceptacles passifs. C'est ainsi que l'on présenta, à la fin du XIV siècle, les Révélations de Brigitte de Suède ou le Dialogue de Catherine de Sienne ${ }^{78}$. Cette symétrie entre un savoir rationnel et analytique

74. Liturgical Life, vol. 1, p. 50 sq.

75. Ibid., p. 52.

76. Ibid. Sur ce point, voir aussi D. H. GREen, Women Readers..., p. 84-115.

77. Voir S. Duval, «Les Dominicains et les femmes. Fin du Moyen Âge - début de l'époque moderne », dans N. Bériou, A. VAucheZ, M. Zink éd., Les Dominicains en France (XIII ${ }^{e}$-XX $X^{e}$ siècle), Paris, 2017, p. 21-38 (p. 33).

78. Sur ce point, $c f$. S. Duval, "Comme des anges sur terre »..., p. 135-136. Sur le cas particulier de Catherine de Sienne, on pourra aussi se reporter à G. Murano, «"Ò scritte di mie mano su l'Isola della Rocca". Alfabetizzazione e cultura di Caterina da Siena », Reti medievali, 18/1 (2017), [en ligne] : http://www.rmojs.unina.it/index.php/rm/article/view/5081 [consulté le 21 septembre 2018]. 
masculin et un savoir « infus » et émotionnel féminin, qui correspond à une grille de lecture cléricale des textes religieux féminins, n'autorisa pas l'accès des femmes au statut d'autorités (auctoritates) du savoir occidental. Elle entraîna toutefois, ou plutôt elle favorisa, l'éclosion à la fin du Moyen Âge d'un grand nombre de prophétesses et de femmes « inspirées » ${ }^{79}$, dont les écrits pouvaient connaître une très grande diffusion ${ }^{80}$.

On relit aujourd'hui ces textes pour y découvrir combien ils sont bien souvent influencés par le savoir universitaire, tout en étant porteurs d'une vision théologique originale. Ainsi, le Dialogue et même les lettres de Catherine de Sienne s'inscrivent dans une veine augustinienne très claire, reflet sans doute de l'influence de ses confesseurs dominicains ${ }^{81}$. On pourra aussi citer à ce sujet le cas très différent de Marguerite Porète et son Miroir des simples âmes, admirablement étudié dans un ouvrage récent ${ }^{82}$. Perçues à travers leurs propres textes, les béguines, tout comme les semi-religieuses italiennes, apparaissent sous un jour différent des portraits, tout en émotions et en prodiges physiques, souvent décrits par leurs confesseurs dans leurs Vitae $^{83}$. On ne se défait donc que peu à peu du filtre imposé par un savoir clérical dominant sur la lecture de textes qui ne lui correspondaient pas pleinement. Rappelons à nouveau ici l'exemple de Maître Eckhart, dont la carrière intellectuelle se développa à l'intersection de ces domaines, puisqu'il fut à la fois un universitaire et un protagoniste remarquable du développement du savoir religieux « alternatif » des religieuses dominicaines dont il avait officiellement la cure (en Alsace, puis en Saxe), mais aussi des béguines. Alain de Libera, dont l'analyse de certains textes des béguines est fondamentale, rappelle qu'il fut condamné pour avoir voulu « trop » savoir, en mêlant le domaine de la théologie spéculative (universitaire) à une approche plus personnelle du divin ${ }^{84}$.

79. Voir notamment A. VAuchez, « Sainte Brigitte de Suède et sainte Catherine de Sienne : la mystique et l'Église aux derniers siècles du Moyen Âge », dans Temi e problemi nella mistica femminile trecentesca, Todi, 1983, p. 229-248.

80. Ce fut notamment le cas pour les Révélations de Brigitte de Suède, diffusées, traduites et abrégées. Voir V. O’Mara et B. Morris éd., The Translation of the Works of St Birgitta of Sweden into the Medieval European Vernaculars, Turnhout, 2000.

81. Voir à ce propos A. VAuchez, Catherine de Sienne. Vie et passions, Paris, 2015, en part. p. 146 .

82. S. L. Field, R. E. Lerner, S. Piron éd., Marguerite Porète et le Miroir des simples âmes, Paris, 2013.

83. V. FRAETERS, «"Ô amour, soit tout à moi !" Le désir comme agent de déification chez Hadewijch de Brabant », dans P. NAGY et D. Boquet éd., Le Sujet des émotions au Moyen Âge, Paris, 2008, p. 324-372.

84. La théologie d'Eckhart se développe ainsi à partir d'un solide savoir scolastique, mais dans une perspective qui le dépasse, car tendue vers la prédication, la cura monialium et donc vers le «savoir » religieux des illitterati (par opposition aux clerici) : A. de LIBERA, Maître Eckhart et la mystique rhénane, Paris, 1999, en part. p. 43-52) ; ID., Penser au Moyen Âge, Paris, 1991, en part. p. 300-330. 
Les études portant sur une période allant du haut Moyen Âge au $\mathrm{XI}^{\mathrm{e}}$ siècle, telles qu'on peut les aborder à travers les deux volumes des Nuns Literacies, présentent un tableau différent. Dans le monde anglo-saxon des $\mathrm{VII}^{\mathrm{e}}-\mathrm{VIII}{ }^{\mathrm{e}}$ siècles, par exemple, on croise dans la correspondance de Boniface, ou bien dans les œuvres d'Aldhelm, de nombreuses moniales et abbesses. Certaines d'entre elles, telle Lioba (qui ne doit pas être considérée comme une exception), furent associées aux missions sur le continent, participant ainsi activement à cet âge d'or de la chrétienté anglo-saxonne ${ }^{85}$. Dans une étude passionnante, Karen Blough retrace les liens culturels ayant existé entre les chanoinesses d'Essen et les moines de Werden (Ruhr) à partir d'un sarcophage du $\mathrm{XI}^{\mathrm{e}}$ siècle représentant des religieuses tenant des livres ${ }^{86}$. Ces contributions présentent toutes, de manière frappante, l'image d'une société ou la moniale cultivée (learned monastic woman) occupait une place de tout premier plan. Cet élément avait déjà été souligné par Rosamond McKitterick, qui s'étonnait que l'on rechignât à attribuer à des religieuses des manuscrits anonymes, sous le seul prétexte qu'elles étaient des femmes ${ }^{87}$.

Cette question est très révélatrice. À partir de quand, et pourquoi se refuse-t-on à attribuer à des femmes l'autorité de textes complexes, ou bien s'ingénie-t-on à minimiser leur rôle dans leur élaboration ? Le Moyen Âge ne considérait sans doute pas de la même manière les auteurs féminins et masculins ; pour autant, les refus d'attribuer à des femmes la pleine responsabilité d'un texte sont caractéristiques de siècles bien postérieurs. C'est au $\mathrm{XIX}^{\mathrm{e}}$ siècle, et jusqu'à très récemment, que l'on a douté de l'authenticité des écrits de Hrotsvita ou d'Héloïse ${ }^{88}$. Aujourd'hui encore, on insiste sur le rôle des clercs et des secrétaires qui entouraient les écrivaines - autant d'éléments qui ne sont en général pas soulignés dans le cas des

85. V. Blanton et H. SCHECK, « Leoba and the Iconography of Learning in the Lives of Anglo-Saxon Women Religious, 660-780 », The Kansas City Dialogue, p. 3-26 ; L. Weston, " Conceiving the Word(s) : Habits of Literacy among Earlier Anglo-Saxon Monastic Women », The Hull Dialogue, p. 149-168.

86. K. Blough, "Implications for Female Monastic Literacy in the Reliefs from St Liudger's at Werden », The Kansas City Dialogue, p. 151-170.

87. R. McKiтterick, "Women and Literacy in the Early Middle Ages », dans EAD., Books, Scribes and Learning in the Frankish Kingdoms, 6th-9th Centuries, Aldershot, 1994, $\mathrm{n}^{\circ}$ XIII, p. 1-43 (p. 24).

88. Voir M. Goullet, « Les drames de Hrotsvita de Gandersheim. Une réécriture dramatique des récits hagiographiques. Approches d'une technique de composition littéraire », Archivum latinitatis medii aevi, 54 (1996), p. 105-130; L. MouLINIER, « H comme Histoire : Hrotsvita, Hildegarde et Herrade, trois récits de fondation au féminin », Clio. Femmes, genre, histoire, 2 (1995), p. 85-107. Sur Héloïse, on se référera à J. Dalarun, « Nouveaux aperçus sur Héloïse et le Paraclet », Francia, 32/1 (2005), p. 19-66, qui, à travers une analyse codicologique aussi précise que passionnante, éclaire autant l'histoire du texte de la « correspondance » que celle de notre propre perception d'Héloïse comme auteure. 
hommes ${ }^{89}$. Ainsi, avant les textes eux-mêmes, ce sont bien nos approches critiques, y compris les plus récentes, qui sont genrées.

Sylvie Duval - Fondation Thiers/CIHAM

culture, écriture, femmes, genre, latin, lecture, littéracie gender studies, Latin, literacy, reading, women, writing

89. Qu'il s'agisse, d'ailleurs, de faire de ce « partenariat » un élément diminuant «l'auteurité » des femmes sur les textes qui leur sont ordinairement attribués, ou bien leur conférant une influence sur des textes qui ne le leur sont pas. Voir à ce sujet la mise au point de J. W. Coakley, Women, Men and Spiritual Power. Female Saints and Their Male Collaborators, New York, 2006. 
